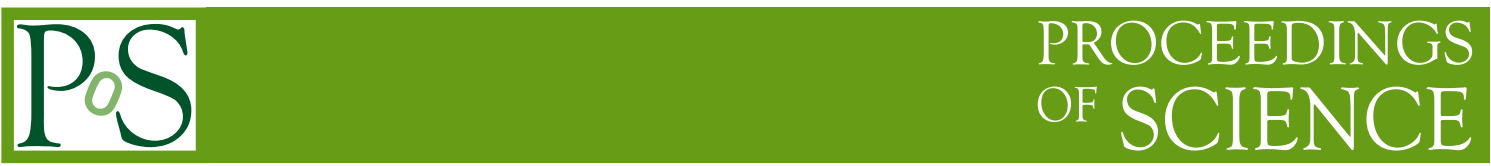

\title{
Coherent elastic neutrino-nucleus scattering
}

\author{
Grayson C. Rich* \\ Enrico Fermi Institute and Kavli Institute for Cosmological Physics, University of Chicago, \\ Chicago, IL, USA \\ On behalf of the COHERENT Collaboration \\ E-mail: gcricheuchicago.edu
}

More than 40 years after its theoretical description, the process of coherent elastic neutrinonucleus scattering (CEvNS) has been observed for the first time by the COHERENT Collaboration, using the world's smallest functional neutrino detector: a 14.6-kg CsI[Na] crystal located at the Spallation Neutron Source of Oak Ridge National Lab. With its observation, CEvNS has emerged as a viable and powerful mechanism for exploring a wide range of physics, with connections to nuclear structure, astrophysics, dark sector physics, and physics beyond the Standard Model. These proceedings discuss the physics accessible with the process and detail aspects of the COHERENT observation. The current and future experimental CEvNS landscape will be surveyed, highlighting the diverse efforts planned and underway within the community, emphasizing their complementarity and underscoring the significant physics reach of this capable, new tool.

Neutrino Oscillation Workshop (NOW2018)

9 - 16 September, 2018

Rosa Marina (Ostuni, Brindisi, Italy)

\footnotetext{
${ }^{*}$ Speaker.
} 


\section{Introduction}

Soon after the experimental confirmation of the existence of the weak neutral current by the Gargamelle experiment, Freedman [1] and the duo of Kopeliovich \& Frankfurt [2] recognized that such a force should allow for the coherent scattering of neutrinos off of nuclei. In such an interaction, the nuclear target remains in its initial state and the coherency of the recoils of the constituent nucleons give rise to a significant enhancement in the cross section. Similar coherent enhancements had already been observed in electron-nucleus scattering [3] and coherent neutrino scattering represented a rather straightforward prediction of the newly confirmed neutral current [1]. Owing to the requirement that the nuclear configuration remain unchanged, and to disambiguate this coherent process from others, e.g. coherent pion production, this process has ultimately been given the more specific name of coherent elastic neutrino-nucleus scattering (CEvNS).

In the decades since its description, theoretical exploration of CEvNS has exposed sensitivity to a wide range of interesting physics, discussed further in Section 2. From its initial prediction, however, the observation of CEVNS stood as an attractive experimental target due to its status as a basic prediction from the Standard Model and its likely relevance to astrophysics: while describing the process, Freedman posited that CEVNS might be significant in core-collapse supernovae (CC$\mathrm{SNe}$ ), participating in the dynamics and driving neutrino opacity [1]. Freedman also presciently observed that an attempt to directly observe the CEvNS process "may be an act of hubris, because the inevitable constraints of interaction rate, resolution, and background pose grave experimental difficulties" [1]. Indeed, despite significant experimental interest, the process would remain unobserved for more than 40 years until the COHERENT Collaboration used a 14.6-kg CsI[Na] scintillator to detect the process for the first time in 2017 [4].

\section{CEvNS physics sensitivities}

The coherent enhancement of the cross section, combined with the very-small weak charge of the proton, results in a characteristic dependence of the CEvNS cross section on the number of neutrons $N$ in the target nucleus, $\sigma \sim N^{2}$. More completely, in the context of the Standard Model and assuming a spin- 0 nuclear target of mass $M$, with $Z(N)$ protons (neutrons), we can express the differential cross section for CE $v$ NS in terms of nuclear recoil energy $T$ as [5]

$$
\frac{d \sigma}{d T}=\frac{G_{F}^{2} M}{4 \pi}\left[N-\left(1-4 \sin ^{2} \theta_{W}\right) Z\right]^{2} F^{2}\left(Q^{2}\right)\left(1-\frac{T\left(M+2 E_{V}\right)}{2 E_{V}^{2}}\right),
$$

with $E_{v}$ the energy of the incident neutrino, $G_{F}^{2}$ the Fermi constant, and $\sin ^{2} \theta_{W}$ the Weinberg or weak mixing angle.

A cursory examination of 2.1 exposes sensitivity to quantities of interest to both nuclear and particle physics. The value of $\sin ^{2} \theta_{W}$ is a fundamental Standard Model quantity, and CE $v$ NS-based measurements of $\sin ^{2} \theta_{W}$ would occur in a region of $Q^{2}$ between the very-low $Q^{2}$ measurement from atomic parity violation (APV) and higher $Q^{2}$ measurements like $Q_{\text {weak }}$ [6]. Sensitivity to nuclear physics is introduced by the presence of the nuclear form factor, $F^{2}\left(Q^{2}\right)$, which encodes information about the spatial distribution of nuclear matter. At low $Q^{2}$, this quantity is indistinguishable from unity; however, at higher values of momentum transfer, complete coherence of the recoil is 
lost and $Q^{2}$ begins to deviate from 1 . Any measurement of $F^{2}\left(Q^{2}\right)$ using CEVNS has enhanced sensitivity to the distribution of neutrons in the nucleus, which represents valuable insight into models for nuclear matter. Using the first data released by COHERENT, Cadeddu et al. were able to extract an average neutron RMS radius for Cs and I of $R_{n}=5.5_{-1.1}^{+0.9} \mathrm{fm}$ [7]. This result for $R_{n}$ can actually be used to make a data-driven correction to the interpretation of Cs APV measurements of $\sin ^{2} \theta_{W}$, as explored in Ref. [8].

Fundamental properties of the neutrino are also accessible with the CEvNS process, including electromagnetic properties such as the neutrino magnetic moment [9] and neutrino charge radii, limits for which were derived from the COHERENT result $[10]^{1}$. Beyond sensitivity to SM physics, CEvNS could be used to probe beyond the Standard Model. Already with the first COHERENT result there have been new limits placed on non-standard interaction (NSI) terms [4], with the process sensitive to new physics such as flavor-changing or non-universal neutral currents [11]. Relating to both NSI and dark-sector physics, CEvNS can be used to remove an ambiguity in oscillation-based determination of the neutrino mass ordering introduced by the LMA-D solution to the octant of $\theta_{12}$ in the presence of NSI [12]. Other dark-sector sensitivities exist, such as the sensitivity to new gauge bosons that kinetically mix with SM photons [13] and other dark $Z$ boson models, potentially explaining the $(g-2)_{\mu}$ anomaly, that would result in a deviation from the SM value for $\sin ^{2} \theta_{W}$ in the region of $Q^{2}$ relevant to $\operatorname{CEvNS}$ [14]. Finally, as a neutral-current process, CEvNS could be used to perform an all-flavor neutrino-disappearance search: an excellent complement to charged-current evidence for the existence of hypothetical "sterile" neutrinos [15].

The relatively large cross section for CEvNS, compared to other neutrino interactions, results in potential significance in a broad array of scenarios. Upon initially describing CEvNS, Freedman [1] recognized that the process could participate in the dynamics of CCSNe; beyond participating in these events, the large cross section means that CEVNS may be a mechanism by which neutrinos from CCSNe could be detected in terrestrial experiments, carrying information about these events that may otherwise be obscured [16]. Another astrophysical connection exists in the realm of particle dark-matter searches: both CEvNS interactions and scattering events with nuclei and dark matter particles produce the same detectable signal in the form of low-energy nuclear recoils; this, combined with astrophysical neutrino sources, means that CEvNS is associated with an irreducible background to many next-generation WIMP searches [17].

\section{Observation of CEvNS with a CsI[Na] detector at the Spallation Neutron Source}

The COHERENT Collaboration was formed with the goal of performing an unambiguous observation of CEvNS using an array of detector technologies/targets and making use of the neutrinos produced at the Spallation Neutron Source (SNS) of Oak Ridge National Lab, near Knoxville, Tennessee, USA. Intended primarily to produce neutrons for material science studies, the SNS operates by bombarding a liquid mercury target with a $\sim 1-\mathrm{GeV}$ proton beam pulsed at $60 \mathrm{~Hz}$, with each beam pulse contained within $\sim 700 \mathrm{~ns}$. In addition to neutrons, the proton beam also produces pions in the target; despite a short lifetime of $\sim 26 \mathrm{~ns}$, a significant fraction of the $\pi^{+}$produced in the target come to rest prior to decaying. This decay at rest yields a population of nearly monoenergetic $v_{\mu}$ which closely follow the timing distribution of the proton beam but also yields $\mu^{+}$;

\footnotetext{
${ }^{1}$ This publication arrived after NOW2018, but is related to the contribution from C. Giunti in these proceedings.
} 
this $\mu^{+}$population subsequently decays (also at rest), yielding a "delayed" neutrino population with a timing distribution that follows the $\mu^{+}$lifetime $(\sim 2.2 \mu \mathrm{s})$ and consisting of both $v_{e}$ and $\bar{v}_{\mu}$ components which have energy distributions well approximated by the Michel spectrum.

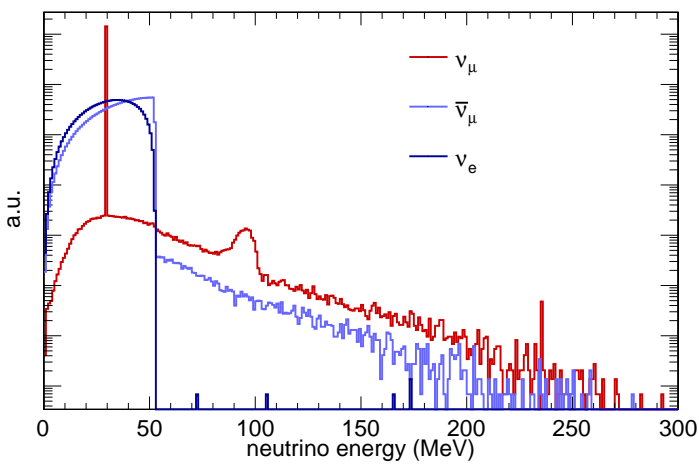

(a) Energy spectra of SNS neutrinos.

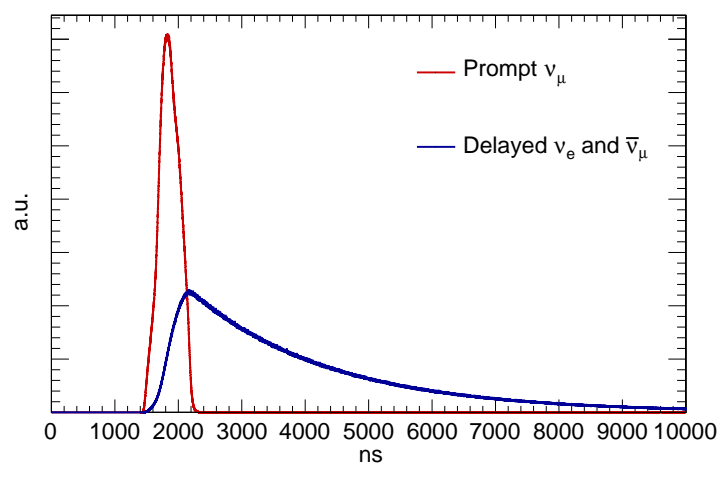

(b) Timing spectra of SNS neutrinos.
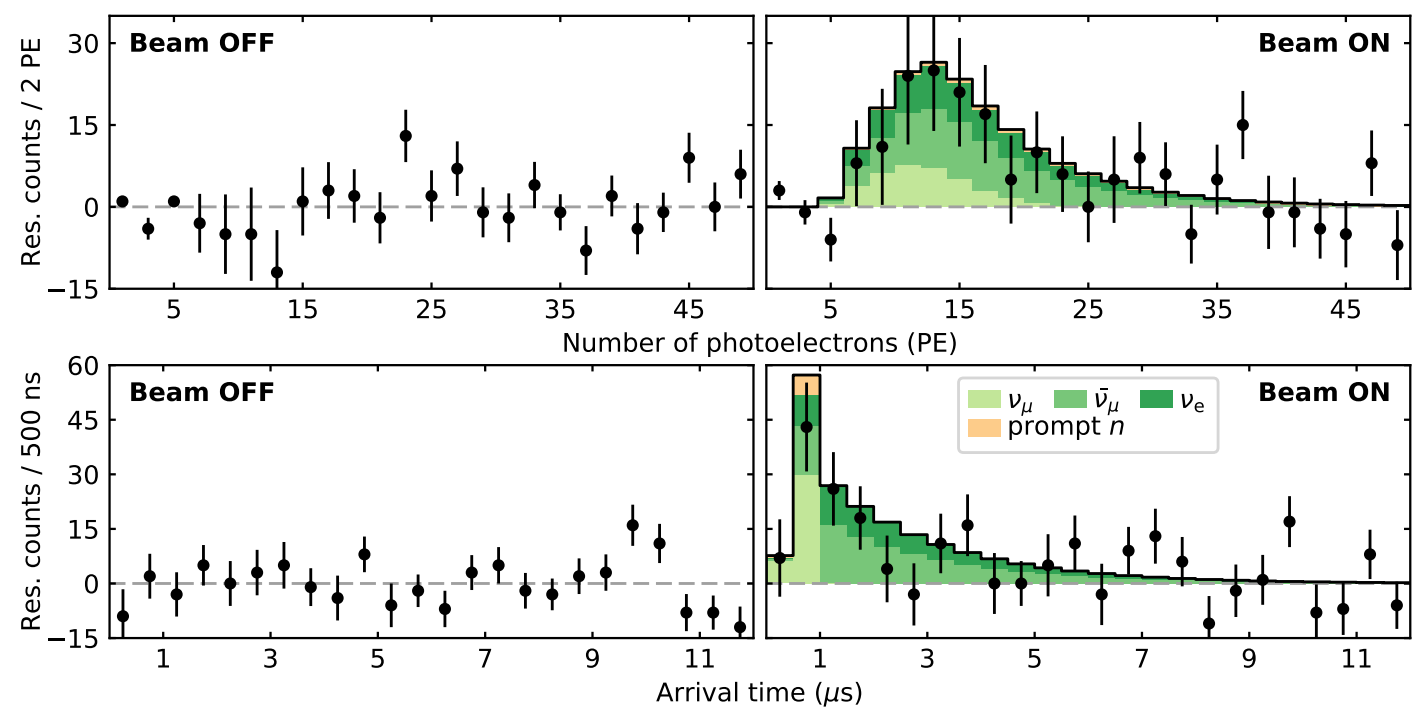

(c) Residual counts from COHERENT CsI[Na] detector.

Figure 1: Neutrino (a) energy and (b) timing spectra for the SNS from a GEANT4 simulation of the liquid-mercury target and an incident $1-\mathrm{GeV}$ proton beam. Prompt and delayed neutrino populations, clearly evident, have distinct energy and flavor compositions. The SNS and relevant details of neutrino production at this source are discussed in the text. Residual spectra in both arrival time and observed energy for the COHERENT CsI[Na] detector are shown in (c), for both beam-off and beam-on periods. The SM CE $v$ NS prediction is overlaid with beam-on residuals. A 2-D profile likelihood analysis showed an observation of CEvNS at the $6.7 \sigma$ level, within $1 \sigma$ of the SM prediction. Figures from Reference [4].

The first detector to be deployed by COHERENT for a CEvNS search was a 14.6-kg CsI[Na] scintillator [18]. Steady-state backgrounds were suppressed by a factor of $\mathscr{O}(1000)$ thanks to the pulsed nature of the SNS neutrino population (see Fig. 1b) and complemented by the modest overburden of $\sim 8$ meters water equivalent available in the so-called "neutrino alley" location, which 
allowed the CsI[Na] detector to be positioned $\sim 20 \mathrm{~m}$ from the liquid-mercury target. A digitizer, triggered by a timing signal from the SNS, recorded 70- $\mu$ s waveforms of the output of a high quantum efficiency ( $\sim 30 \%)$, super-bialkali PMT viewing the CsI[Na] crystal in addition to the output of a high-efficiency muon-veto system. Beam-coincident and -anticoincident regions were defined in the waveforms, with the anticoincident region informing a model for the steady-state background found in the coincident region. Performing a 2-D profile likelihood analysis in both time and energy space, the COHERENT Collaboration reported an observation of the CEvNS process with a significance of $6.7 \sigma$, consistent with the Standard Model prediction within $1 \sigma$ [4].

\section{The CEvNS experimental landscape}

There is an exciting and robust collection of experimental efforts focused on CEvNS, with present and near-term deployments mapped in Fig. 2. COHERENT ${ }^{2}$ continues to operate the CsI[Na] detector at the SNS with data collection likely to be discontinued in 2019. The collaboration has also deployed and is collecting CEvNS data with a single-phase liquid argon (LAr) detector, CENNS-10, with a fiducial volume of $\sim 22 \mathrm{~kg}$ for the physics run. Future plans include an array of germanium p-type point contact (PPC) detectors with a mass of $\sim 10 \mathrm{~kg}$, a 2-ton array of $\mathrm{NaI}[\mathrm{Tl}]$, a ton-scale LAr detector, and a $\mathrm{D}_{2} \mathrm{O}$ detector to perform a direct $v$-flux measurement.

Outside of COHERENT, the planned CEvNS experiments will utilize $\bar{v}_{e}$ from nuclear reactors: though the flux of these neutrinos can be considerably higher, the energies are lower and the detectors must be capable of very-low energy thresholds. Numerous capable technologies have been selected for this purpose. The CONNIE collaboration uses silicon CCDs with a target threshold of 28-eV ionization energy [19]. Germanium PPCs, targeted for use by COHERENT, CONUS ${ }^{3}$ [20], TEXONO [21], and $v \mathrm{GeN}$ [22] have been shown to be capable of ionization-energy thresholds of $<300 \mathrm{eV}$ [20]. PPC technology was advanced significantly by the WIMP-search community, which also contributed to the development of dual-phase xenon detectors like that used by RED-100 [23] for CEvNS detection and the Si and Ge detectors designed originally for SuperCDMS but planned for use in the MINER CEvNS effort [24].

The NU-CLEUS ${ }^{4}$ and Ricochet experiments will make use of bolometric techniques to achieve exceptionally low thresholds. Ricochet plans to use Ge semiconductor detectors as well as superconducting $\mathrm{Zn}$ devices, aiming for recoil-energy thresholds of approximately $50 \mathrm{eV}$ [25]; NUCLEUS intends to use $\mathrm{CaWO}_{4}$ and $\mathrm{Al}_{2} \mathrm{O}_{3}$ devices, having demonstrated a $\sim 20-\mathrm{eV}$ recoil-energy threshold in the latter [26]. The BASKET project represents another bolometric approach at an earlier R\&D phase, seeking to develop $\mathrm{Li}_{2} \mathrm{WO}_{4}$ scintillating bolometers [27].

With the robust experimental program that has emerged in the CEVNS arena, it is important to emphasize the complementarity that exists between many of these diverse experiments. One example of the complementarity between $v$ sources of different energies is the ability to isolate nuclear physics effects in a specific nuclear target when data exists at several values of $Q^{2}$. Additionally,

\footnotetext{
${ }^{2}$ For brevity, some aspects of the COHERENT physics portfolio that are not directly associated with CEvNS are omitted.

${ }^{3}$ For additional information on the CONUS experiment, see the NOW2018 contribution from M. Lindner

${ }^{4}$ The NU-CLEUS experiment is discussed in more detail by the NOW2018 contribution from J. Rothe.
} 


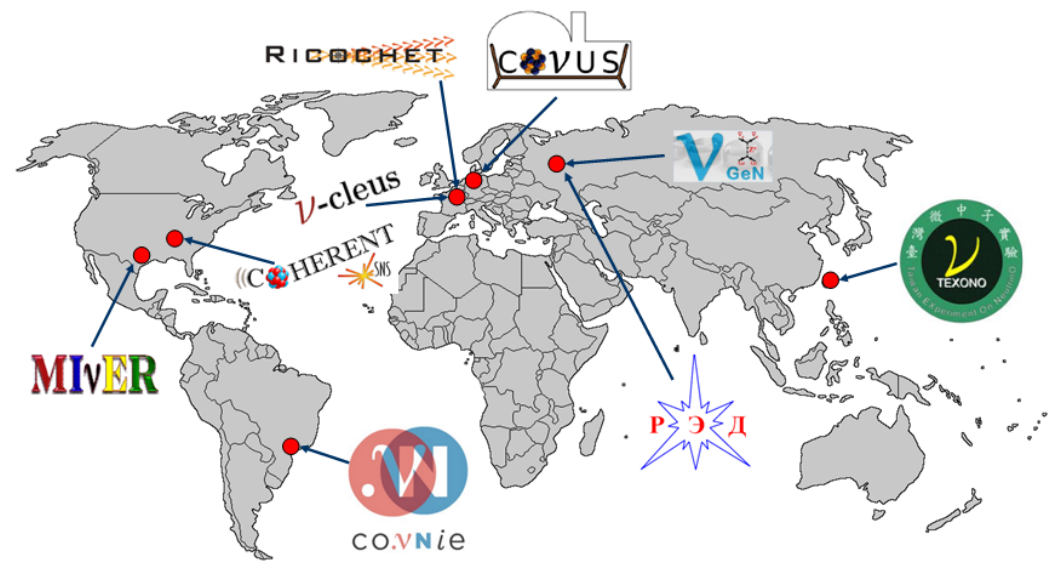

Figure 2: World CEvNS efforts collecting data or with near-term plans for deployment. With the exception of COHERENT, all experiments will be located at nuclear reactors: the MINER experiment is to be sited at a research reactor on the campus of Texas A\&M (US) [24]; CONNIE is located near the Angra II reactor (Brazil) [19]; NU-CLEUS [26] and Ricochet [25] are exploring deployment at the Chooz reactor complex (France); both RED-100 [23] and $v \mathrm{GeN}$ [22] are planned for the Kalinin Nuclear Power Plant (Russia); CONUS [20] is at Kernkraftwerk Brokdorf (Germany); and TEXONO [21] is at the Kuo-Sheng nuclear power station (Taiwan). Figure from Alexey Konovalov.

several studies have found significant enhancements to the sensitivity to neutrino NSI, in particular, when combining results from different target nuclei and different neutrino sources [28, 29].

\section{Conclusions}

Observed for the first time in 2017, more than 40 years after its prediction, CEvNS is a powerful tool that can be used to explore a broad range of physics. Its connections to nuclear physics, astroparticle physics, and to fundamental properties of neutrinos and the Standard Model have motivated a vibrant and diverse experimental community, with the variety of experiments made more valuable by the significant complementarity that exists between CEvNS measurements made with different nuclear targets and neutrino sources.

The author is supported in part by the Kavli Institute for Cosmological Physics at the University of Chicago through an endowment from the Kavli Foundation and its founder Fred Kavli. Numerous other funding sources supported the work of the COHERENT Collaboration and are acknowledged in Akimov et al. [4].

\section{References}

[1] D. Freedman, Coherent effects of a weak neutral current, Phys. Rev. D 9 (1974) 1389.

[2] V. Kopeliovich and L. Frankfurt, Isotopic and chiral structure of neutral current, JETP Lett. 19 (1974) 145. 
[3] R. Hofstadter, Nuclear and nucleon scattering of high-energy electrons, Annu. Rev. Nucl. Sci. 7 (1957) 231.

[4] D. Akimov, J. Albert, P. An, C. Awe, P. Barbeau, B. Becker et al., Observation of coherent elastic neutrino-nucleus scattering, Science 357 (2017) 1123 [1708.01294].

[5] M. Lindner, W. Rodejohann and X.-J. Xu, Coherent neutrino-nucleus scattering and new neutrino interactions, J. High Energy Phys. 2017 (2017) 97 [1612 . 04150 ].

[6] B.C. Cañas and E.A. Garcés and O.G. Miranda and A. Parada, Future perspectives for a weak mixing angle measurement in coherent elastic neutrino nucleus scattering experiments, Phys. Lett. B 784 (2018) 159 [1806.01310].

[7] M. Cadeddu, C. Giunti, Y. F. Li and Y. Y. Zhang, Average CsI Neutron Density Distribution from COHERENT Data, Phys. Rev. Lett. 120 (2018) 072501 [1710. 02730].

[8] M. Cadeddu and F. Dordei, Reinterpreting the weak mixing angle from atomic parity violation in view of the Cs neutron rms radius measurement from COHERENT, Phys. Rev. D 99 (2019) 033010 [1808.10202].

[9] T. Kosmas, O. Miranda, D. Papoulias, M. Tórtola and J. Valle, Sensitivities to neutrino electromagnetic properties at the TEXONO experiment, Phys. Lett. B 750 (2015) 459 [1506.08377].

[10] M. Cadeddu, C. Giunti, K. A. Kouzakov, Y. F. Li, A. I. Studenikin and Y. Y. Zhang, Neutrino charge radii from COHERENT elastic neutrino-nucleus scattering, Phys. Rev. D 98 (2018) 113010 [1810.05606].

[11] J. Barranco, O. Miranda and T. Rashba, Probing new physics with coherent neutrino scattering off nuclei, J. High Energy Phys. 2005 (2005) 021 [hep-ph/ 0508299 ].

[12] P. Coloma, M. C. Gonzalez-Garcia, M. Maltoni and T. Schwetz, COHERENT enlightenment of the neutrino dark side, Phys. Rev. D 96 (2017) 115007 [1708.02899].

[13] S.-F. Ge and I. M. Shoemaker, Constraining photon portal Dark Matter with TEXONO and COHERENT data, J. High Energy Phys. 2018 (2018) 66 [1710.10889].

[14] H. Davoudiasl, H.-S. Lee and W. Marciano, Muon $g-2$, rare kaon decays, and parity violation from dark bosons, Phys. Rev. D 89 (2014) 095006 [1402.3620].

[15] A. Anderson, J. Conrad, E. Figueroa-Feliciano, C. Ignarra, G. Karagiorgi, K. Scholberg et al., Measuring active-to-sterile neutrino oscillations with neutral current coherent neutrino-nucleus scattering, Phys. Rev. D 86 (2012) 013004 [1201.3805].

[16] C. Horowitz, K. Coakley and D. McKinsey, Supernova observation via neutrino-nucleus elastic scattering in the CLEAN detector, Phys. Rev. D 68 (2003) 023005 [astro-ph/ 0302071 ].

[17] J. Billard, E. Figueroa-Feliciano and L. Strigari, Implication of neutrino backgrounds on the reach of next generation dark matter direct detection experiments, Phys. Rev. D 89 (2014) 023524 [1307.5458].

[18] J. Collar, N. Fields, M. Hai, T. Hossbach, J. Orrell, C. Overman et al., Coherent neutrino-nucleus scattering detection with a CsI[Na] scintillator at the SNS spallation source, Nucl. Instrum. Methods Phys. Res. A 773 (2015) 56 [1407. 7524].

[19] G. Fernandez Moroni, J. Estrada, E. E. Paolini, G. Cancelo, J. Tiffenberg and J. Molina, Charge coupled devices for detection of coherent neutrino-nucleus scattering, Phys. Rev. D 91 (2015) 072001 [1405.5761]. 
[20] W. Maneschg et al., The Status of CONUS, in Neutrino 2018, June, 2018, DOI.

[21] A. Soma, M. Singh, L. Singh, G. K. Kumar, F. Lin, Q. Du et al., Characterization and performance of germanium detectors with sub-keV sensitivities for neutrino and dark matter experiments, Nucl. Instrum. Methods Phys. Res. A 836 (2016) 67 [1411.4802].

[22] V. Belov, V. Brudanin, V. Egorov, D. Filosofov, M. Fomina, Y. Gurov et al., The vGeN experiment at the Kalinin Nuclear Power Plant, J. Instrum. 10 (2015) P12011.

[23] D. Akimov, A. Berdnikova, V. Belov, A. Bolozdynya, A. Burenkov, A. Dolgolenko et al., Status of the RED-100 experiment, J. Instrum. 12 (2017) C06018.

[24] G. Agnolet, W. Baker, D. Barker, R. Beck, T. Carroll, J. Cesar et al., Background studies for the MINER Coherent Neutrino Scattering reactor experiment, Nucl. Instrum. Methods Phys. Res. A 853 (2017) 53.

[25] J. Billard, R. Carr, J. Dawson, E. Figueroa-Feliciano, J. A. Formaggio, J. Gascon et al., Coherent neutrino scattering with low temperature bolometers at Chooz reactor complex, J. Phys. G Nucl. Partic. 44 (2017) 105101 [1612.09035].

[26] R. Strauss, J. Rothe, G. Angloher, A. Bento, A. Gütlein, D. Hauff et al., Gram-scale cryogenic calorimeters for rare-event searches, Phys. Rev. D 96 (2017) 022009 [1704. 04317 ].

[27] M. Vivier, C. Nones and A. Zolotarova, Development of innovative cryogenic detectors for precise measurement of coherent elastic neutrino-nucleus scattering, in Neutrino 2018, June, 2018.

[28] J. B. Dent, B. Dutta, S. Liao, J. L. Newstead, L. E. Strigari and J. W. Walker, Accelerator and reactor complementarity in coherent neutrino-nucleus scattering, Phys. Rev. D 97 (2018) 035009 [1711.03521].

[29] J. Billard, J. Johnston and B. Kavanagh, Prospects for exploring new physics in coherent elastic neutrino-nucleus scattering, J. Cosmol. Astropart. Phys. 2018 (2018) 016 [180 5 . 01798]. 